\title{
Using the computer driven VR environment to promote experiences of natural world immersion
}

\author{
Lisa A. Frank*a \\ Beckman Institute of Advanced Science and Research, University of Illinois Urbana-Champagne, \\ 405 North Mathews Avenue, Urbana, IL USA 61801; \\ Wisconsin Institutes for Discovery, University of Wisconsin-Madison, \\ 330 N. Orchard Street, Madison, WI, USA 53715
}

\begin{abstract}
In December, 2011, over 800 people experienced the exhibit, $<1>$ : "der"//pattern for a virtual environment, created for the fully immersive CAVETM at the University of Wisconsin-Madison. This exhibition took my nature-based photographic work and reinterpreted it for virtual reality (VR).Varied responses such as: "It's like a moment of joy," or "I had to see it twice," or "I'm still thinking about it weeks later" were common. Although an implied goal of my 2D artwork is to create a connection that makes viewers more aware of what it means to be a part of the natural world, these six VR environments opened up an unexpected area of inquiry that my 2D work has not. Even as the experience was mediated by machines, there was a softening at the interface between technology and human sensibility. Somehow, for some people, through the unlikely auspices of a computer-driven environment, the project spoke to a human essence that they connected with in a way that went beyond all expectations and felt completely out of my hands. Other interesting behaviors were noted: in some scenarios some spoke of intense anxiety, acrophobia, claustrophobia-even fear of death when the scene took them underground. These environments were believable enough to cause extreme responses and disorientation for some people; were fun, pleasant and wonder-filled for most; and were liberating, poetic and meditative for many others. The exhibition seemed to promote imaginative skills, creativity, emotional insight, and environmental sensitivity. It also revealed the CAVETM to be a powerful tool that can encourage uniquely productive experiences. Quite by accident, I watched as these nature-based environments revealed and articulated an essential relationship between the human spirit and the physical world. The CAVETM is certainly not a natural space, but there is clear potential to explore virtual environments as a path to better and deeper connections between people and nature. We've long associated contact with nature as restorative, but those poetic reflections of Thoreau and others are now confirmed by research. Studies are showing that contact with nature can produce faster, greater recovery from stress and other illnesses, reduction in anger, and an increased sense of well-being. Additionally, I discovered that the novelty of a virtual reality experience can bring new focus and fresh attention to elements of our world that we have grown immune to. Possibly, the 'boletus edulis' in one scene seemed to have been made more remarkable and mysterious in VR than if it was seen in the backyard. A VR environment can be used to create opportunities to experience being in the world differently. Here they can be inside of an egg that is inside of a nest that is held by tree branches over a creek bed in a floating landscape where a light spring snow is falling. We are liberated from the worldly limitations of our body. The question is this: in an anti-natural environment, can immersants in a CAVETM become more ecologically sympathetic and spiritually connected? Although the exhibit was not put through any form of testing as of yet, my observations amount to a remarkable vision of what VR might provide for us as an instrument to expand consciousness and promote wellness. Creating exceptional, transformative experiences may seem like a lofty goal for VR but that purpose is at the heart of any art making process.
\end{abstract}

Keywords: $\mathrm{CAVE}^{\mathrm{TM}}$, nature, virtual reality, exhibition, art, imagination, perception, photography

1.

\section{INTRODUCTION}

The CAVETM art exhibition, $<1>$ : “der"//pattern for a virtual environment, used a CAVETM in a visually expansive way that went beyond its conventional purposes. This exhibition was experienced by over 800 visitors during the course of ten evenings in December, 2011. The project provides a lively example of what is possible when artistic concerns form a partnership with science and technology in an exploratory, multi-disciplinary pursuit, and signals the promise of rich and remarkable collaborations. ${ }^{1}$

*alafrank@mac.com; phone 1222 555-1234; lisafrankphotography.com 
In this paper I will focus on the following themes: my path to the CAVE exhibit including a description of my artwork, its references, and the process involved in reinventing my artwork for a virtual reality space; my exploration of the CAVETM's potential for producing immersive aesthetic experiences and the discoveries and questions that evolved from that effort including observations about the powerful process of collaboration. I will also discuss the use of immersive environments for the purpose of exploration and reconnection with nature.

\subsection{Artist's statement}

The CAVETM exhibition is composed of six different virtual reality scenes that were based on pre-existing digital photography. In this first section I will describe the original artwork as well as the process involved in preparing and interpreting the work for the CAVE ${ }^{\mathrm{TM}}$.

The origin of the exhibit's imagery begins far away from the CAVETM's projection screens. In my art-making practice, I explore themes related to environment, nature, pattern, and place. Over the past five years, I have been making largescale photographs constructed from the thousands of snapshots that I take while walking in the woods. As these photographs are pieced together, complex patterns take form and a personal, arbitrary time chart is revealed. This activity of taking pictures as I walk - in all seasons, in all weather - makes for a visual journal that documents changing evidence of the ordinary and the astonishing. All things are equal. I am as captivated by the full and magnificent bloom of color as I am to the existential challenges implicit in its fade and decay.

Densely ornamental, the patterns reference the interior decoration documents of Britain's Arts and Crafts Movement of the late 19th century. The designer, William Morris, is particularly relevant. His wallpaper designs drew upon similarly themed subject matter, showing a love for all natural things and designing with an assurance gained from observing nature first hand.

My artwork is connected to the "wildness" in nature while taming it with formal patterning. The inter-relationships between nature's corresponding parts form the true "patterns" of my work. Creating a context for connection, it is my purpose to draw the viewer into a local world as it hasn't been seen before - charged with wonder - rich, complex and changeable while being inclusive and accessible.

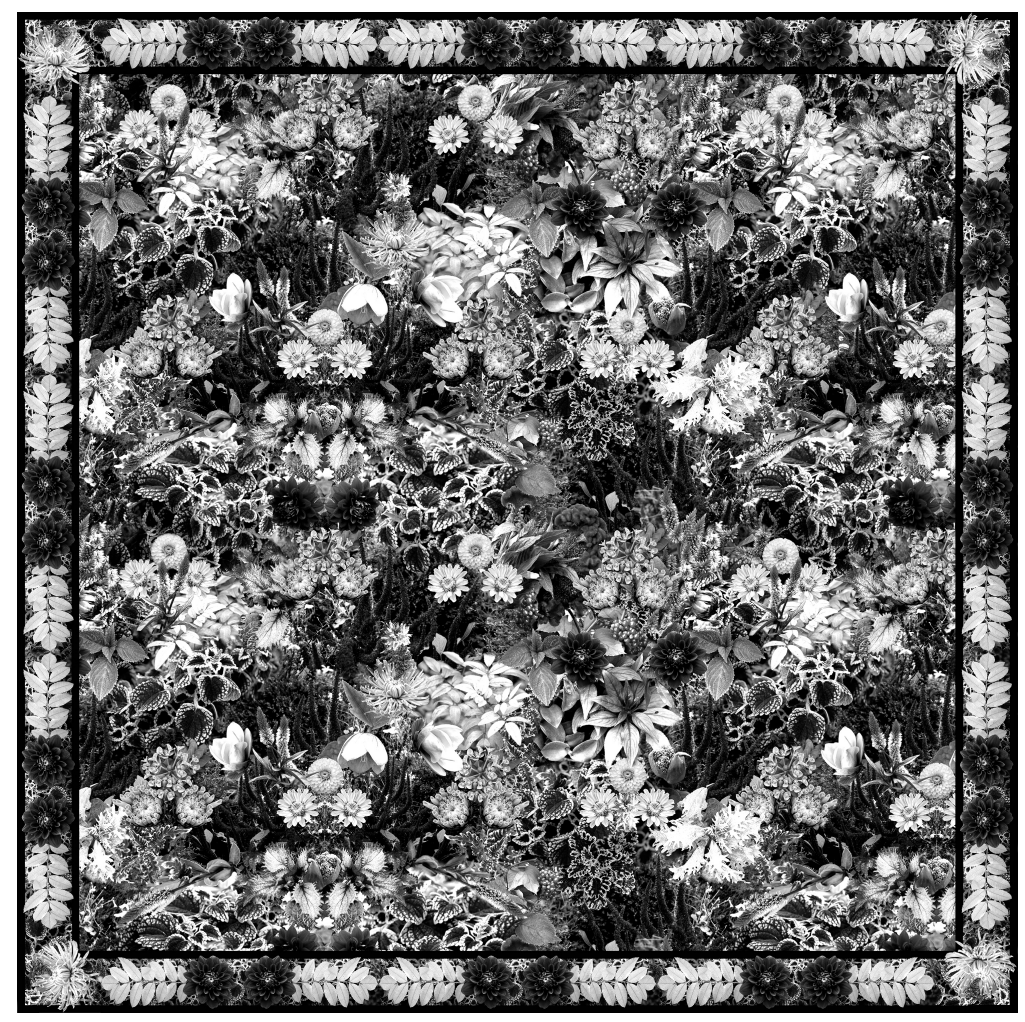

Figure 1. "Early Autumn Floral," a digital photograph by Lisa A. Frank (2010). 


\subsection{How it happened}

As a thesis opportunity in 2011, I was presented with a challenge: what could I do with my two-dimensional artwork that would push it in an unexpected direction. It was suggested that I create an exhibition using the University of WisconsinMadison's new CAVETM facility. Although completely unfamiliar with the history, practice or potential of virtual reality applications I quickly realized that the CAVE ${ }^{\mathrm{TM}}$ could push my two-dimensional studies of nature and pattern into a new future. Here was an opportunity for decorative patterns to be fully immersive, created as walk-about environments where the viewer could explore the whole 'and' its parts: moving elements about, changing scale and perspective, living within their shifting spaces and then floating outside their realm. In an evolving 'theater' space, the viewer could become part of and partner to the scenery. Instead of deceiving only the eye, as with a trompe l'oiel painting, the CAVE ${ }^{\mathrm{TM}}$ could potentially deceive the entire self or whole groups of people, and open a world of creative, technical, scientific, and aesthetic opportunities with that deception.

\subsection{The exhibition}

Each of the six fully immersive scenarios, or "dioramas," present a unique visual and spatial composition. If I break down any one of these to its smallest component, it would be a single photograph taken while walking in the woods. The scenarios are informally labelled 'Nest,' 'Rabbit Room,' 'Milkweed,' 'Bleeding Heart,' 'Mushroom,' and 'Bloodroot,' after their primary subject matter. Participants view the scenes one directly after another in a prescribed order. To view the exhibition in its entirety involves a minimum of 15 minutes.

All of my artwork - 2D or 3D - considers early natural history painting as an important reference. Before the advent of photography artists and botanists alike created botanical paintings. Working directly from nature, subjects seemed keenly observed but were often imaginatively interpreted. Blending myth and observation with varied levels of artistry, what was unknown or not understood by the artist/scientist could simply be fabricated.

I, too, keenly observe but imaginatively interpret. I am astounded by the oddity and fancy of the natural world and am driven to invent stories that might explain the origins of some curious species. The odd life cycle of the Jack-in-thePulpit, for example, makes its way into several of the scenes, along with falling purple tulip petals that match the color and shape of the plant's strange hood. In the tradition of Rudyard Kipling's, Just So Stories, ${ }^{2}$ a highly improbable codependency is inferred. (video link ${ }^{3}$ )
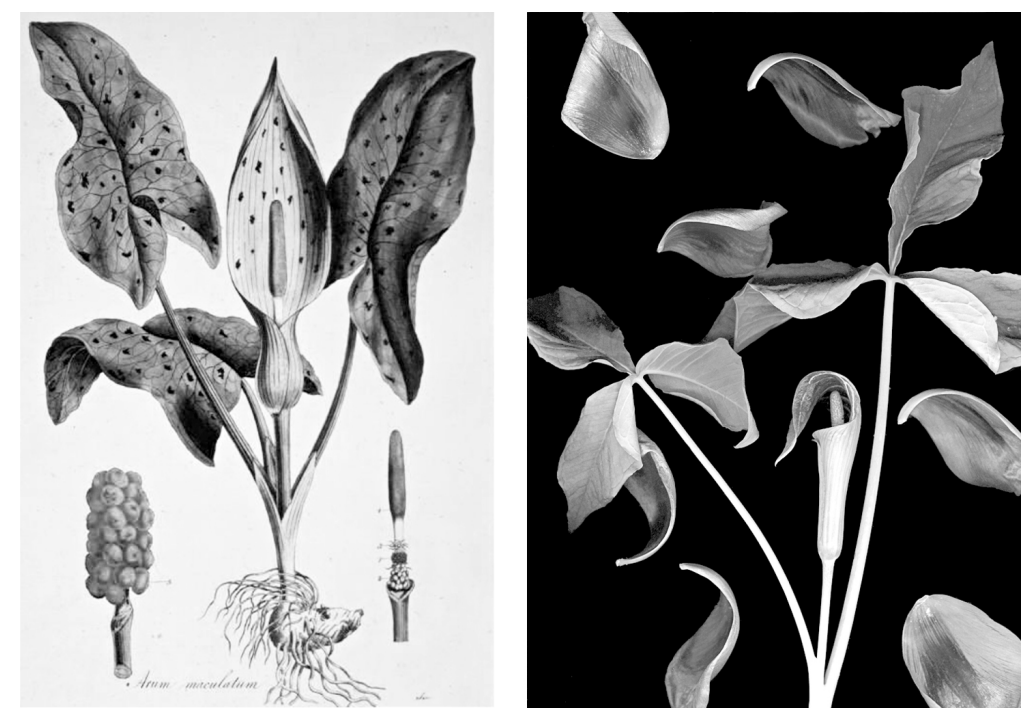

Figure 2. "Arum macalatum” (“Lords and Ladies”) by William Curtis, from Flora Londinensis, (1777-1787).

Figure 3. "Jack-in-the-Pulpit with Tulip Petals," a digital scanograph by Lisa A. Frank (2007).

In the CAVETM's virtual space, this fictional-factual, topsy-turvy world can spring to life. 'Immersants' are given the unique opportunity to fly over treetops. They crouch inside a nest, find themselves in an egg, break through the egg's crack, and swim out into a liquid landscape. Immersants watch electrified 'mycelia' at work under the forest floor and stand at dirt level beneath prodigiously 'wild' mushrooms. They negotiate a path through towering woodland plants and look down on a mosaic floor cobbled together with rocks from the last ice age. (video link ${ }^{4}$ ) 


\subsection{The instrument}

The primary instrument used for the exhibition was a CAVETM immersive visualization system with a fully enclosed sixsurface configuration (9'6" x 9'6" x 9'6") built in 2011.

The CAVETM $\mathrm{C} 6$ is constructed as a room with four walls (one opens fully as a door), a ceiling, and a plexiglass floor. All six sides of the CAVETM are projection screens. Behind each projection screen are two projectors which combine to form 3D rear projected images on each screen. The system also has a 5.1 surround sound audio system and ceiling microphones for two-way communication. The C6 uses 'Intersense' head and wand tracking for full user-viewpointdependent stereoscopic viewing. Ultrasonic tracking sensors embedded into the CAVETM corners provide full tracking of the head and wand.

The CAVETM virtual reality system allows multiple users (a maximum of 8 ) to experience the virtual environment at the same time. The viewers explore the environment by moving around inside the cube.

The computers are running Windows XP 64-bit. The underlying display technology is the 'CAVELib' application programmer interface. Most of the exhibition has been rendered in 'OpenSceneGraph' (OSG), using 'GLSL' shaders to control movement and animated effects.

The CAVETM is used as a research tool by the 'Living Environments Laboratory' (LEL) at the Wisconsin Institutes for Discovery (http://discovery.wisc.edu/lel). The thrust of LEL's research lies in the use of advanced visualizations and information architectures to improve human performance in real and virtual environments.

\subsection{Collaboration: The art of productive collisions}

The trend of interdisciplinary collaboration is becoming increasingly evident. Recent studies have shown that conversations across multiple disciplines - where concepts get transposed, recombined, and compounded - can prove to be uniquely fertile. While the cross-pollination of many types of science and research is itself an extraordinary step, results can be even more animated and insightful when connections and unusual pairings rise up beyond the traditional domain of the sciences.

Central to my experience preparing artwork for the CAVE ${ }^{\mathrm{TM}}$ was the essential collaboration with computer scientists, particularly my assistant, Nathan Mitchell and a software engineer from Mechdyne Corporation, Andrew Cherry.

I worked for several months with Andrew as the CAVETM was under construction. He developed some preliminary, experimental code for me and offered strategies for how I might set up my images. In September, 2011, Nathan Mitchell, a computer sciences graduate student at the University of Wisconsin-Madison, took up the reins as my assistant for the next four months. Our collaborative relationship was unlike any I've had in the past.

Nathan and I came from very different worlds and as collaborators we needed to mentor each other every step of the way. He grew up with 3D graphics, computer games, and joysticks. Seeing the movie, "Avatar," was the full extent of my digital 3D exposure. We had different languages: I would say "form," and he would say "geometry." Recognizing this, we spent several weeks familiarizing each other with the visual worlds that we each knew. I showed Nathan the wallpaper designs of William Morris and he showed me many computer games (particularly insightful was the wildly colorful "Alice: Madness Returns").

I had ponderous goals. I wanted to create something that would offer nothing short of a fully transformational experience; however, I had no idea how to make such a thing. In the beginning of our collaboration Nathan said something that had a profound effect on me, "We can probably do anything you can think up." Although this could be understood as free-wheeling potential, it only magnified my state of "cluelessness." I consoled myself with the thought that whenever one changes mediums, there is a learning curve while technical skills are developed, techniques are experimented with and mistakes are made. I told myself "one semester is not enough time to master much of anything. What could I possibly pull off in a few months without a mentor, a manual, or a model? I was truly in the dark.

The overarching questions for weeks were: "Where is this headed (and why)? How will I get there? How much work is it going to involve? and What will I end up with?" No one could answer these questions, or most others, in a reassuring or definitive way. Documentation that could offer practical insights and successful strategies was simply not available. This made me well aware of the possibility that it could become a failed effort.

Nathan and I spent the first 2 months grappling with technical issues: How do I get the sharpest, truest image from such a small number of pixels? How many files can the system handle without crashing? How do I take my complicated 2 GB photoshop files and turn them into something the CAVETM technology can make sense of? During the last weeks before the exhibition, after months had been invested in the process of learning new tools and technology, through much 
trial and error while developing an unusual partnership, I began to catch a glimpse of the expressive and experiential potential for future work in the CAVETM. Importantly, I was no longer afraid of coming up empty-handed. The work displayed for the exhibition went as far as was possible in four months.

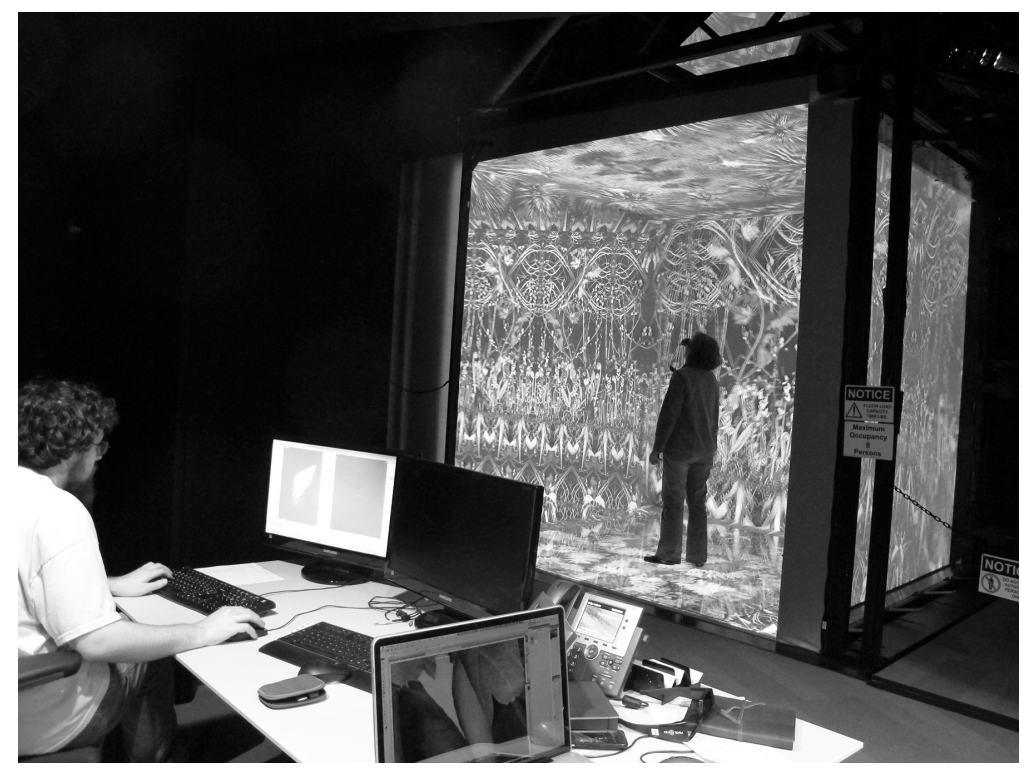

Figure 4. Working on the exhibition with Nathan Mitchell, University of Wisconsin-Madison CAVETM in 2011.

2.

BREAKING IT DOWN

As I began to consider my approach for choosing which of my 2D pieces to explore in 3D I looked at a few references. My 2D work is conceived for the wall, it implies an environment and an architecture. But the CAVE ${ }^{\mathrm{TM}}$ makes that environment go haywire because the walls aren't stable. In the real world, patterned wallpaper is simply put up and then later, taken down, while the CAVETM advances more complex activities.

\subsection{The panoramic view}

I considered the photographic idea of the 'panorama', defined as a picture or series of pictures that represent a continuous and complete scene, often an all-encompassing overview. "The panoramic mirage was most potent in the stereoscope, in its 'appearance of reality', as Oliver Wendell Holmes put it, which cheats the senses with its seeming truth. I pass, in a moment, from the banks of the Charles to the ford of the Jordan and leave my outward frame in the arm-chair at my table, while in spirit I am looking down on Jerusalem from the Mount of Olives." 5 The stereoscope, and later the View-Master, gave the general public a travel experience to far-away lands. This stereoscopic form of sightseeing - whether to the Eiffel Tower or the Great Wall of China - was made available through a photographic trick.

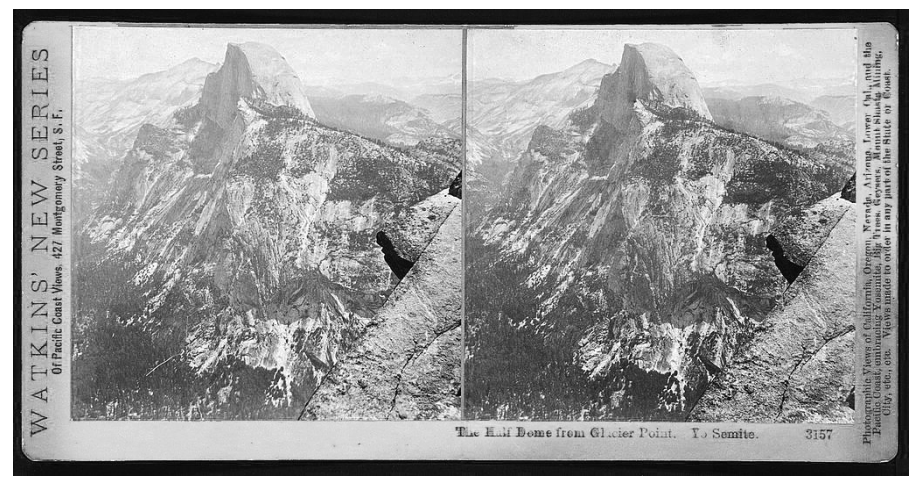

Figure 5. "The Half Dome from Glacier Point, Yosemite"; stereograph published by C. Watkins; (1876-79). 
I connected with the idea of creating a spatial illusion that was analogous to an actual panorama. I remembered my childhood experiences of looking at views of Yosemite, Old Faithful, and the Grand Canyon on View-Master reels - all made into dimensional, magical, expansive space right before the mind's eye. With a click of the reel, I could happily transport myself to these natural "wonders of the world." This memory provided me with some direction. I fixed myself on the goal of capturing "wonderment" in an immersive, illusionistic, walk-about experience.

\subsection{Virtual reality references}

I familiarized myself with the immersive virtual spaces created by abstract painter, Char Davies, in the 1990's. Davies redefines 'immersive virtual space' as a "means for de-habituating perception and re-sensitizing us to our own being in the world." She approached the medium as an arena for "changing space" as meant by the French philosopher and essayist, Gaston Bachelard, who wrote in The Poetics of Space, "By changing space, by leaving the space of one's usual sensibilities, one enters into communication with a space that is psychically innovating...For we do not change place, we change our Nature." 7

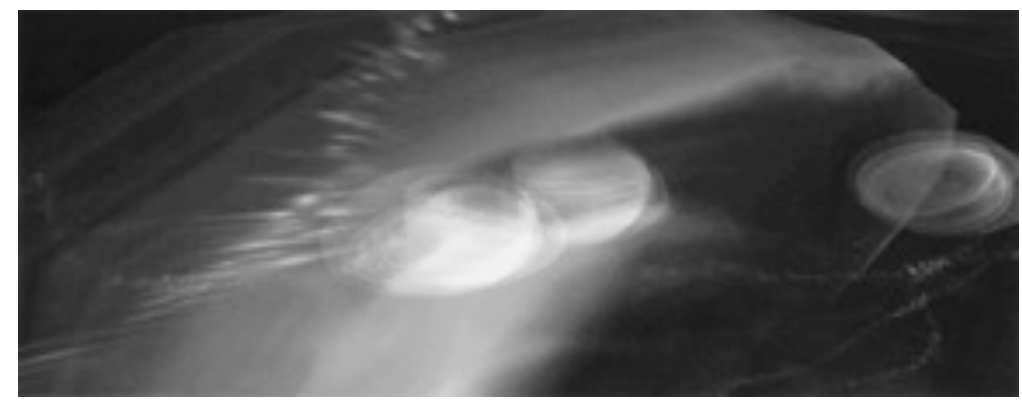

Figure 6. "Eggs in the Interior Body" from Ephémère, by Char Davies. Digital still captured in real-time through HMD (1998). ${ }^{8}$

Even though her work was created for a head-mounted display (HMD), seeing still images of her two major works, Osmose and Ephémère, strengthened my sense that there is great potential for using a CAVE ${ }^{\mathrm{TM}}$ as an expressive art medium. Her virtual environments are archetypal realms of nature ('Forest, Leaf, Clearing, Pond, Cloud and Abyss').They are semi-transparent, abstract and fluid spaces, ephemeral rather than representational, recalling the atmospheric paintings of J.W. Turner. The spaces are completely illusionistic.

In "Art of the Digital Age," Davies is quoted: "My goal is to use immersive virtual space to collapse the boundaries between Subject and Object, Interior and Exterior, and Self and Other. In doing so, my intent is to refresh our perception of being in the world. I want to resensitize participants to the extraordinariness of being alive, sentient and embodied, here now among all this, immersed as we are so briefly in the flow of life through space and time." challenge of using VR technology alternatively, as an antidote that reaffirms our embodied participation within the natural world rather than our instrumentally objectifying conquest of it.

3.

COMPELLING NATURE

A rich paradox is revealed. Virtual reality can be experienced as an artificially constructed symbolic space, but also, paradoxically, as an actual space which feels real in the sense of being a dimensionally extended, all-surrounding, navigable environment. In such an environment, participants can move about and explore as they choose, encompassed by spatial potentialities, as if it really were an actual place. Davies writes, "Such paradox (such slippage of boundaries between the virtual and the actual, between pre-constructed concept and experientially-flowing percept - whereby the medium's immateriality can be experienced as coexisting with an apparently real three-dimensionality of form, whereby it is possible to seemingly float gravity-free among semi-transparent entities and pass through them as well." 10

\subsection{Paradoxical nature}

Immersive virtual space, when approached unconventionally, can provide a channel through which we can perceive freshly what we 'think' we know - that mushrooms, for example, grow under trees. The paradoxical quality of the medium - that the mushrooms in the CAVE ${ }^{\mathrm{TM}}$ are in fact, no where near a tree - allows us to perceive what we have grown immune to, free from our daily distractions and assumptions. 


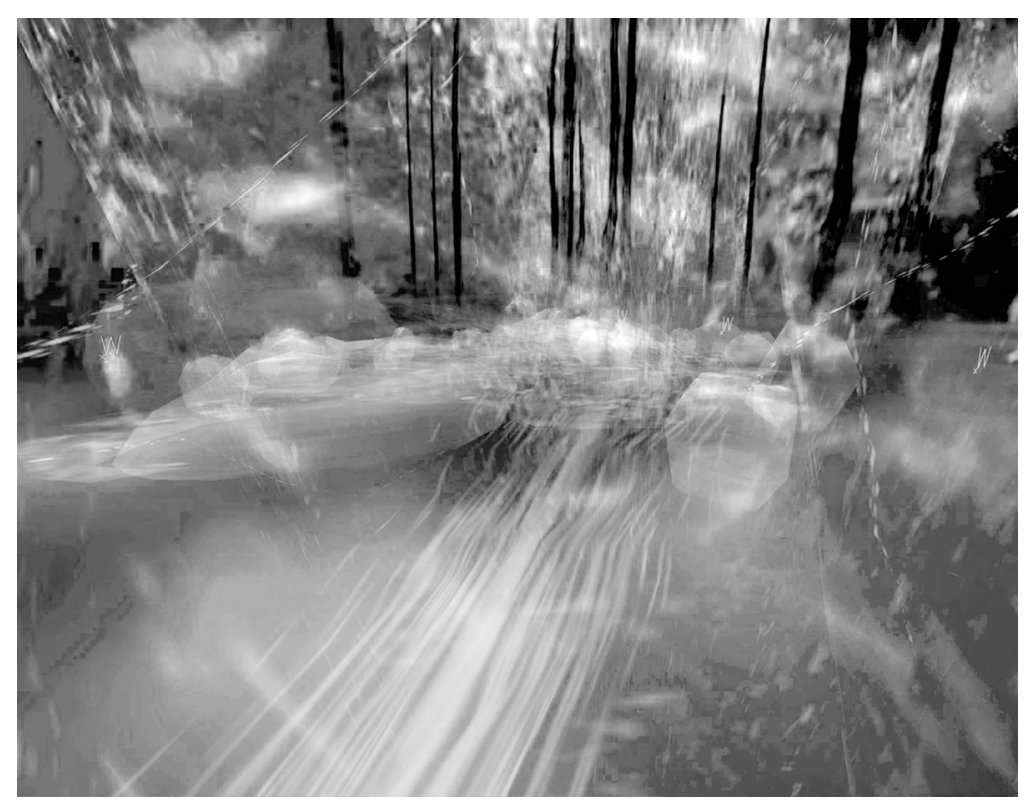

Figure 7."Forest Stream" from Ephémère by Char Davies. Digital still captured in real-time through HMD during live immersive journey/performance (1998). ${ }^{11}$

The CAVETM project seemed to make people aware of things that they had become numb to, information that they had discarded--particularly, that we are a part of, not separate from nature. In the CAVETM the ordinariness of a plant is powerfully transformed and re-discovered in virtual reality more so than if that same plant was discovered in the backyard. Oddly, the advanced technologies used in these exhibitions do not seem to threaten our connection to the physical world - instead they offer possibilities for reconnection to it.
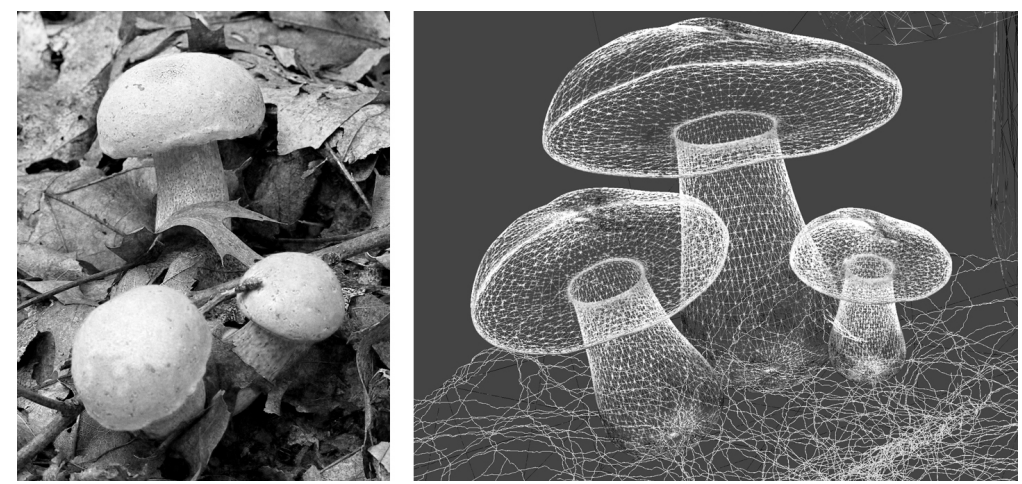

Figure 8. Digital photograph of wild mushrooms (2011).

Figure 9. Screenshot of wire mesh model used in the "Mushroom scenario" (2011)

\subsection{Unconventional nature}

Gordon Orians, renowned ornithologist, maintains that our attraction to nature exists at the level of our DNA: "The biological world, like the mental world of Ebenezer Scrooge is replete with ghosts. There are ghosts of habitats, predators, parasites, competitors, mutualists, and conspecifics past, as well as ghosts of meteors, volcanic eruptions, hurricanes, and droughts past. These ghosts may reside in our genetic attic, but sometimes they speak to us, whispering the past is present". ${ }^{12}$

I have used imagery in the exhibition that has traceable repetition. As in nature, patterns are everywhere, even if they only register subconsciously over the course of fifteen minutes. The Milky Way's spiral is represented, as is the macro world of the 'boletus edulis' and the micro life of mycelia. 
The CAVETM experience took a tacit goal of my 2D artwork - to make observers more ecologically aware of what it means to be in the world - and expanded those possibilities, making it, potentially, a consciousness-producing instrument. It is through this immersive experience that one might come to the conclusion that this human-made virtuality of a natural world may eventually become all we have left to remind us of the deep forest and the way it feels to be alive in that particular world.

4.

\section{OBSERVATIONS}

Creating artwork for the CAVETM has also become linked for me with the study of human emotions in their widest and wildest sense. Even as the experience is mediated by machines, there is a softening at the interface between technology and human sensibility. Somehow, for some people, through the unlikely auspices of a computer-driven environment, the project speaks to a human essence that they connect with, in a way that feels completely out of my hands.

\subsection{Art and art therapy}

"What I am trying to translate to you is more mysterious; it is entwined in the very roots of being, in the impalpable source of sensations." 13 (J. Gasquet, Cézanne)

The feedback was rich and fascinating and it opened up an area of personal inquiry that was unexpected. I was frankly startled by the response it received. Why did a young girl say that it was "like a moment of joy?" Why did a 19-month old baby squeal with delight throughout, reaching and grabbing for whatever she was perceiving, surprising everyone with her animated response? Why did an older woman describe the 15 minutes as "staying with her" like "the best kind of movie," one that she came back again to see? Another said, "I'm still thinking about it after weeks have passed!" These responses are very different than the ones my 2D work provokes - they were animated and uninhibited and spoken by a wider and more diverse audience.

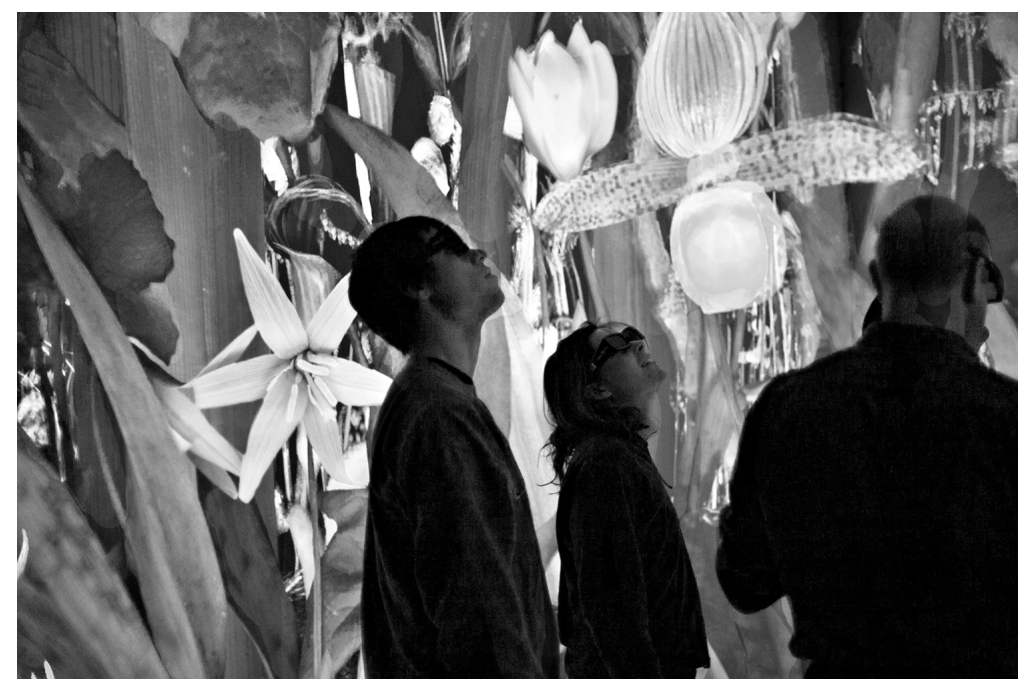

Figure 10. Participants in the "Bloodroot scenario," University of Wisconsin-Madison, 2011.

Studying viewers' emotional responses seems most closely linked to art and art therapy. The wide variation of responses and behaviors evoked by the CAVE ${ }^{\mathrm{TM}}$ exhibit led me to many questions worth additional research. Why did some people report intense anxiety in the nest because it actually engaged their acrophobia? Why was this environment believable 'enough' that it could cause such an extreme fear response for some people, be pleasant and liberating for most, and disorienting for others? How convincing does something have to be in the CAVETM for 'most' to suspend their disbelief?

In the 'Mushroom Scenario' there were again a wide variety of responses, this time to the idea of being underground. For some it was a fun, "ant's eye-view" experience while for others it engaged their claustrophobia and a fear of being buried alive, or even their fear of death. It clearly is a tool to potentially draw out both positive and negative responses. 


\subsection{Perception}

"Our experience of the world is an interpretation based on the strategies we developed for making decisions about what to focus on.Although the CAVE ${ }^{\mathrm{TM}}$ may simply be projection arts, it still requires an interpretation of a confrontation within the real world."

As some participants in the Nest scenario felt themselves flying over the scene, soaring through scattering leaves, looking down upon the nest they were just standing in, others could only observe the artificial environment but not feel in any way part of it. The environment is noticeably believable enough that the two participants in the video link actually peer over the edge of the nest at the creek below; but when they reach out to catch the fluttering leaves, what is it that they are really grasping? (video link ${ }^{15}$ ) This seems to articulate an essential relationship between the human spirit reaching out for something we can not hold - and the physical world.

In Through the Vanishing Point: Space in Painting and Poetry, Marshall McLuhan wrote, "One of the peculiarities of art is to serve as an anti-environment, a probe that makes the environment visible." He goes on to state that the role of the artist is to "open the door of perception to people otherwise numbed in a non-perceivable situation." He emphasized that this function of art is betrayed if the artist "merely repeats the bias of the culture instead of readjusting it"16

Immersive virtual spaces can be used to convey alternative sensibilities and world views.Viewers are immersed in an alternative environment, experiencing an event that does not physically exist in the so-called real world. These experiences are completely illusory; they are, in effect, the theatre of the mind. ${ }^{17}$

In the "new world view" of the exhibition, we are eyeball-deep in dirt and roots. In this illusionistic space we are no more or less impressive than dirt, but instead we are equal to it. This provided a clue for how to tackle the question of the CAVETM's unique potential and how to understand its effects. (video link ${ }^{18}$ )

\subsection{Imagination}

The creation of imaginative spaces not only gives us the opportunity to experience an "other" type of "being" in the world, it represents a new existence that is both real and virtual. We can be inside an egg that is inside a nest that is up in the leaves of a tree. We can become a speck of dirt, we can stand inside a birdcage, we can feel ourselves cut in half by a rogue leaf. We recognize ourselves differently, if only momentarily. In virtual worlds "there is both a fluidity and speed of movement that are more akin to dreams than waking life,"19 and so can the content be.

Beyond the aesthetic and immersive opportunities the CAVETM provides, there seems to be rich potential for exploring and encouraging imagination. In virtual worlds "there is both a fluidity and speed of movement that are more akin to dreams than waking life." 18 The creation of imaginative spaces not only gives us the opportunity to experience an "other" type of "being" in the world, it represents a new existence that is both real and virtual. There exists the capacity to traverse environments quickly or slowly, fly in space, cross walls and barriers and interact with or touch objects in a manner never before possible. Concepts can be reversed. As creators we are liberated from the worldly limitations of our bodies.

In Engineering Nature, Art and Consciousness in the Post-Biological Era, Roy Ascott writes, "It is still unknown what impact virtual reality experiences may have on the imagination and on the development of human consciousness...To date,VR technologies have seen relatively widespread applications in education, research and medicine, therapy and rehabilitation, business and design and - especially - entertainment. On the other hand, very little investment has been made in VR applications that are designed to promote imaginative skills such as aesthetic sensitivity, reflection, artistic creativity or emotional insight." 20

5.

METHODOLOGY

At the time of the exhibition in December, 2011, I lacked the foresight that my work would touch upon research areas that could benefit from formal studies. As a result I did not have a strategy in place that would formally question and monitor immersants' experiences during and directly after their participation in the exhibition. I have since become aware that the exhibition, and evolving work, could serve as a research tool to tease out information related to imagination, creativity, and general wellness issues. Due to the expense of running the CAVE ${ }^{\mathrm{TM}}$ at the University of Wisconsin-Madison there has not been the opportunity during this past year to revisit the exhibition for the purpose of conducting specific studies. 


\subsection{Surveys in a hurry}

I was, however, able to put together 2 small surveys for the purpose of this paper. The first survey was answered by 15 college educators who were guests of the University in November, 2012. The second survey "CAVE Exhibition: One Year Later," was sent in December, 2012 to 45 people, aged 35-70, who had experienced the exhibition one year before.

It was answered by 23 who first stated that they had very clear memories of the exhibition. Both groups were more homogenous in composition than the larger range of visitors to the original exhibition in 2011. The table below lists some of the results from the two surveys.

Table 1. Results from two recent surveys.

\begin{tabular}{|l|l|l|}
\hline \multicolumn{1}{|c|}{ Statement } & \multicolumn{1}{|c|}{$\begin{array}{c}\text { One hour later } \\
(\mathbf{1 5} \text { results) }\end{array}$} & \multicolumn{1}{|c|}{$\begin{array}{c}\text { One year later } \\
\text { (23 results) }\end{array}$} \\
\hline I had fun. & $80 \%$ & $100 \%$ \\
\hline I felt a sense of wonderment at least once. & $92.8 \%$ & $100 \%$ \\
\hline I felt like I was transported somewhere else at least once. & $80 \%$ & $81.8 \%$ \\
\hline The exhibition was inspiring. & $80 \%$ & $100 \%$ \\
\hline As a result of the exhibition I am in a better mood. & $60 \%$ & $90.9 \%$ \\
\hline I felt uninhibited during the exhibition. & $80 \%$ & $57.2 \%$ \\
\hline As a result of the exhibition I feel less anxious or worried. & $60 \%$ & $86.3 \%$ \\
\hline The exhibition made me think about nature in new ways. & $50 \%$ & $72.7 \%$ \\
\hline I felt like I was falling at least once. & $64.3 \%$ & $60 \%$ \\
\hline I felt like I was flying at least once. & $73.4 \%$ & $55 \%$ \\
\hline If you had the opportunity, would you go through this exhibition again? & $80 \%$ & $100 \%$ \\
\hline
\end{tabular}

\subsection{Survey results}

The most convincing results were in the following areas. 100\% of the "One Year Later" group agreed or strongly agreed with the statement, "I felt a sense of wonderment at least once." $82 \%$ agreed or strongly agreed with the statement, "I felt like I was transported somewhere else at least once." $100 \%$ agreed or strongly agreed that, "The exhibition was inspiring."

It was evident during the project that immersive virtual art environments were a very new experience for the majority of visitors, which has a definite emotional and cognitive impact. Visitors took pleasure in the immersive experience with most being excited to explore the exhibition again. Although the majority of responses commented that it was moderately to extremely easy to navigate and move about the exhibit, it was commonly stated that the exhibition made them feel sick and/or dizzy occasionally. Some remarked that they felt at times lost or confused. Mostly, the results revealed it as a technology that encourages a powerful and unique experience for the user - more accessible, more fun, more playful, and more reflective than a traditional computer-mediated approach.

6.

CONCLUSIONS

We've long associated contact with nature as being restorative and reflective. But many of the poetic reflections by Henry David Thoreau and others are confirmed by scientific process. The research of environmental psychologists, Rachel and Stephen Kaplan, Roger S. Ulrich, and others has indicated that contact with nature, especially vegetation, has a beneficial effect on physical and psychological health (in the sense that nature both calms and focuses the mind).

\subsection{Ecotherapy}


Ulrich demonstrated through several different studies that contact with nature produced faster and greater recovery from stress as shown by lower blood pressure, less muscle tension, reduction in anger and an increased feeling of well-being. Not surprisingly, these are many of the same beneficial effects found through a contemplative practice. With that in mind, future VR experiences could potentially be conceived as contemporary landscapes for contemplation, emulating the powers of healing gardens ("horticultural therapy"), therapeutic spaces, and labyrinths. With the intention of unfixing ourselves from our usual world view, it shares a common practice with meditation, chanting, dance (and the ingestion of hallucinogenic mushrooms!).

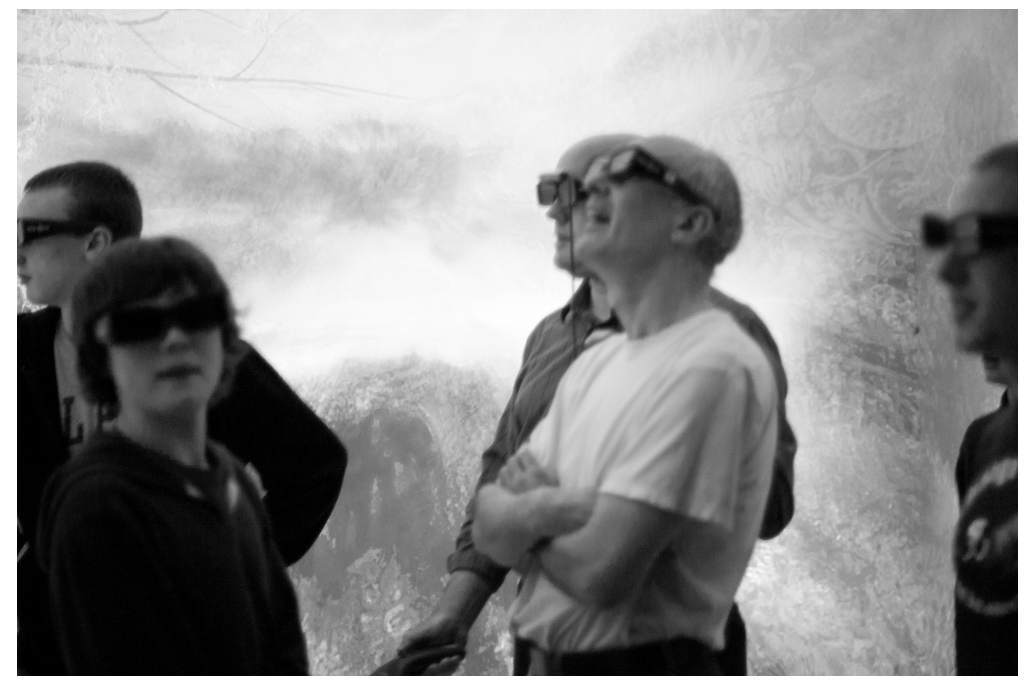

Figure 11. Participants in the "Nest scenario," University of Wisconsin-Madison, 2011.

Additional research supports the contention that "nature therapy" helps control pain and negative stress. For people with heart disease, dementia, and other health issues, the "nature prescription" has benefits that may go beyond the predictable results of outdoor exercise. The restorative power of the natural world can help us heal, even at a relative distance.

Building on this point, studies have shown that hospital patients with views of trees have shorter hospital stays. Another study shows that patients who were simply shown a mural of a mountain stream in a spring meadow with a continuous tape of complementary nature sounds had substantially better pain control than those without. ${ }^{22}$ It's not a stretch to consider that a CAVETM experience of nature could be effective in treating patients who are not able to have an out-ofdoors experience themselves but who could physically navigate a virtual out-of-doors. In this way it becomes a potentially viable 'stand-in': a high-tech mural, a technological tonic, a nature-cure.

Running counter to the traditional ethos of computers, VR could be harnessed as a unique tool capable of affecting positive change in the areas of health, wellness, restoration and reconnection. It becomes a context for exploring how to make ourselves whole within a technological frame.

Robert Louv shares an important theory that "the best preparation for the 21 st century may be a combination of natural and virtual experience, with the ideal pilot being the person who has a balance of high-tech and natural knowledge. We need people who have both ways of knowing the world. A hybrid mind." 23

\subsection{The artist informing VR}

In her book, Computers as Theatre, Brenda Laurel writes, "For virtual reality to succeed in meeting these goals, we need continual and deep involvement by artists in the ongoing process of understanding what virtual reality is for and what it can be. We need convivial tools that allow artists to work in the medium in order to influence its evolution. Most of all, we need artists to help us understand how virtual reality, like other art forms, can inform and enrich human experience." 24

With this is mind I'd like to return to the subject of collaboration and explore how the insights and scholarship of an artistic perspective could inform the research goals of virtual reality, encouraging both more aesthetic applications of the technology, but also more effective research. A scientist might consider an idea put forth by an artist as irrelevant, far- 
fetched, and off-subject, but it is also possible that the scientist might 'then' find - two days later - that the same idea transposed into a more familiar language, becomes a fascinating, missing piece.

Inspiration can be found in the artist-in-residence program at the Xerox Palo Alto Research Center (video link) ${ }^{25}$ where scientists and artists who use the same new media or media tools informed and explored each others' work. Or, in the collaboration between choreographer Liz Lerman and the Wesleyan University Life Sciences program, now called "Science Choreography," where faculty, researchers, students, and dancers explored new ways to see, feel, and understand science. As the overview describes it: "The project changed us all in ways we had not predicted: The scientists found new ways to think about and teach science, and the dancers came to understand more about how the scientists see the world." 26

I believe there is true potential in blending art, science, humanities, their sub-disciplines into a complex fabric not only because we share similar threads but because we are so often dramatically different. The problems we are confronting today are immensely complicated. At the cutting edge of new knowledge, environments that encourage the exploration of diverse interests, expertise and insights will generate solutions that rise up from the resonance and tension between their fields of view. I see the artist invaluably "leaning into" these discussions, and agree with Brenda Laurel who writes, "Our creative force must be manifest, not as an artifact but as a collaborator-an extension of ourselves embodied in our systems. There must be more behind the looking glass than a room that one steps into, and there must be more to virtual reality than the engineering of the looking glass." 27

\subsection{Unusual and rare}

There are unfortunate realities regarding the accessibility of CAVETM technology: it's expensive and it's uncommon. I have also discovered that not all "CAVETM's are "created equal." A CAVETM that is one year old has many improvements over one that is 10 years old. The age of projectors can vastly affect aspects of the exhibit that I have spent a great deal of time focused on such as color saturation, clarity, lightness and darkness. The newer CAVETM at the University of Wisconsin shows the outcome of this work clearly. The same work projected in the older CAVETM at the University of Illinois looks dark and muddy in comparison, the subtleties and hard work are undetectable.

To encourage both aesthetic applications and effective research, navigational issues could benefit from future enhancements. Many visitors report some physical discomfort in immersive environments which corrupts their potential experience and creates results that are uneven and skewed. Certainly there will always be people who will find 3D viewing physically intolerable, but providing most participants with a navigational experience that is stabilized and controlled would produce better results. When the surveyed group of educators went through the exhibition in November, as an example, I personally controlled the joystick (which I usually don't). Not surprisingly, this group reported more physical discomfort than other groups. Due to some "performance anxiety" issues, I tend, unfortunately, to use the joystick in a faster and jerkier way. Since it's hard to create a positive experience if physical symptoms interfere, it would be beneficial to simply have options that could smooth and control the speed of navigational movement.

\subsection{Potential}

In closing, responses to the exhibit, $<1>$ : "der"//pattern for a virtual environment, confirm that the CAVETM and other virtual reality systems are ripe with potential for discovery. The novelty of a virtual reality experience can bring new focus and fresh attention to elements of our world that we have become resigned or mute to. "The power of immersive environments lies in the fact that these environments are psychological thresholds that have the potential to encourage new perspectives" 28 This amounts to a remarkable vision of what virtual reality might provide for us.

"What happens when one steps inside the magic circle?" The magic nest? The magic egg? "The meaning of the ordinary is transformed." ${ }^{29}$ The CAVETM is certainly not a natural space, but there is clear potential to explore virtual environments as a path leading to better and deeper connections between people and nature. Can it urge more contact with nature? Can immersants become more ecologically sympathetic and spiritually connected through a machine? Can it make people more aware of what they have become immune to? The art exhibition, $<1>$ : "der"//pattern for a virtual environment, creates a context for new connections with the natural world. It demonstrates ways that our perspectives can be refreshed, inciting us to be alive in the natural world, differently. Creating exceptional, transformative, aesthetic experiences may seem like a lofty goal for VR but that purpose is at the heart of any art making process. 


\section{REFERENCES}

[1] Brooks, S., "CAVE of wonders: Exhibit pushes the boundaries of art, collaboration" http://www.news.wisc.edu/ 20118, 9 December 2011.

[2] Kipling, R., [Just So Stories, Illustrated Edition]; Yesterday’s Classics, Chapel Hill, NC; (2009).

[3] Frank, L. "Lisa A. Frank: Exhibit in the CAVE," video link: http://www.youtube.com/watch?v=OjE7eQc6vl8, 8 December, 2011.

[4] Frank, L., "Wonder: Pattern for a Virtual Environment; Making the Exhibition: Part 1: Artist's Statement," video link: http://www.youtube.com/watch?v=gCeIPi49Lpk, 8 December, 2011.

[5] Trachtenberg, A., Reading American Photographs: Images as History, Hill and Wang, New York, 85, (1989).

[6] Davies, C. "Virtual Space," in "Space: In Science, Art and Society," Cambridge University Press, Cambridge, UK, 69-204, 2004.

[7] Bachelard, G., The Poetics of Space (La Poétique de l'Espace), Translated by Maria Jolas, Beacon Press, Boston, MA, 206, (1964).

[8] McRobert, L., [Char Davies' Immersive Virtual Art and the Essence of Spatiality], University of Toronto Press, Toronto \& Buffalo \& London, C14 illus., (2007).

[9] Wands, B.. [Art of the Digital Age], Thames \& Hudson Inc., New York, 28-30, (2006).

[10] Davies, C., "Virtual Space," Space: In Science, Art and Society, Cambridge University Press, Cambridge, UK, 69-104, (2004).

[11] Davies, C., "Virtual Space," Space: In Science, Art and Society, Cambridge University Press, Cambridge, UK, 69, illus., (2004).

[12] Louv, R., [The Nature Principal], Algonquin Books of Chapel Hill, Chapel Hill, NC, 53, (2012).

[13] Merleau-Ponty, M.,"Eye and Mind," (Joachim Gasquet's "Cezanne: A Memoir With Conversations”), Gallimard, Paris, (1960).

[14] Dolinsky, M., "Collaborative Virtual Environments Art Exhibition,” Proc. SPIE-IS\&T Electronic Imaging 5664 , 641-652, (2005).

[15] Frank, L., "Sam and Dayna in the Nest," video link: http://www.youtube.com/watch?v=N7QJebqS-Sk, 21 May, 2012.

[16] McLuhan, M., [Through the Vanishing Point: Space in Painting and Poetry], HarperCollins, New York, 241, (1968).

[17] Shanken, E., [Art and the Electronic Media], Phaidon Press Ltd., London, 172-195, (2009).

[18] Frank, L.,"Nathan's Forest: Wild Mushrooms," video link: http://www.youtube.com/watch?v=DJMSWlnZTNw, 7 January, 2012.

[19] Dolinsky, M., "Collaborative Virtual Environments Art Exhibition," Proc. SPIE-IS\&T Electronic Imaging 5664 , 641-652, (2005).

[20]Ascott, R., [Engineering Nature, Art and Consciousness in the Post-Biological Era] (2006).

[21] Louv, R., [The Nature Principal], Algonquin Books of Chapel Hill, Chapel Hill, NC, 52-55, (2012).

[22] Louv, R., [The Nature Principal], Algonquin Books of Chapel Hill, Chapel Hill, NC, 47, (2012).

[23] Louv, R., [The Nature Principal], Algonquin Books of Chapel Hill, Chapel Hill, NC, 39, (2012).

[24] Laurel, B., [Computers as Theatre], Addison-Wesley Publishing Company, Inc., Reading, MA, 196, (1993).

[25] Xerox Palo Alto Research Center (PARC) website video: http://www.parc.com/services/focus-areas.html)

[26] "Science Choreography," website, video: http://sciencechoreography.wesleyan.edu, (8 April 2011).

[27] Laurel, B., [Computers as Theatre], Addison-Wesley Publishing Company, Inc., Reading, MA, 196, (1993).

[28] Castricano, J., "Testing the Limits": What Happens When Digital Humanities Meets Alternative Worldviews," Digital Sudies / Le champ numerique; Vol. 2, No 2 (2011).

[29]Laurel, B., [Computers as Theatre], Addison-Wesley Publishing Company, Inc., Reading, MA, 197, (1993). 